\title{
Interactive comment on "A study of polarimetric noise induced by satellite motion: Application to the 3MI and similar sensors" by Souichiro Hioki et al.
}

\section{Otto Hasekamp (Referee)}

o.hasekamp@sron.nl

Received and published: 3 November 2020

This paper investigates the polarimetric error that results from non-instantaneous acquisition by different polarization filters, representative for filter-wheel based instruments such as $3 \mathrm{MI}$ and the POLDER-1,-2, and -3 instruments. It has been suggested for long time in the polarimetric remote sensing community that this may be an important source of error and I'm glad to see that the authors quantify this error term. Also, the authors show that the magnitude of the polarimetric error may be predicted using the along-track Laplacian, available from the measurements themselves. This 
a number of comments which are important but quite easy to address. I recommend publication of the paper after addressing the comments.

\section{Comments}

- It would be better to use the term 'error' instead of noise because it is not a purely random error (see also comment of other reviewer). Also, I suggest to clearly quote values for bias and RMSE as measures of accuracy. The 5-95 percentile is also useful but the inter-quartile range is less relevant. Probably here the 15.9-84.1 percentile range is more useful as it would represent the standard deviation for a Gaussian error distribution.

- Please quote the bias and RMSE for all 4 categories in the abstract.

- Which wavelength has been used for this study? Was it 670 or 860 nm? For clear-sky over land I would expect the error at $860 \mathrm{~nm}$ is (much) larger than at $670 \mathrm{~nm}$. I would recommend to show errors at both wavelengths.

- Please make clear that the resulting errors are on L1B product. Further processing to L1C may introduce extra errors (Lang et al., JQSRT, 2019), but not necessarily related to the measurement acquisition approach.

- Please relate the errors to the total error budget of 3MI. Is it a dominant error source?

- How do these errors relate to errors on aerosol and cloud properties. For aerosols, we have investigated the relation between DoLP accuracy and retrieval accuracy (e.g. Hasekamp et al., JQSRT, 2019). Not sure if a similar study exists for cloud retrieval.

- Also the case of clear-sky over ocean would be interesting. Probably the errors would be small here (at 670 and $860 \mathrm{~nm}$, maybe not at 400-500 nm) but that would also be important information for users. I suggest to add that case.

- Figure 6 shows the median and 'spread' of the error as function of $L$ at/L. Can the median be interpreted as the median of the DoLP_diff distribution corresponding to a 
given value of $L \_a t / L$ ? I would expect this is a bin of $L$ at $/ L$ values, otherwise you would have very few points to compute the mean and spread. Or am I mis-interpreting the AMTD figure? How is spread defined here? - Why are there large parts in Fig 7 'Gray hatched' (statistics unavailable or unreliable) but not in Fig. 6?

- The authors show that the noise found from SGLI for the cloud over water case is similar to the noise found for a radiance field simulated for random cloud distributions following a power law distribution. They call this 'prediction of noise'. I would say this is not the 'prediction of noise/error', but rather an 'understanding of error'. The prediction of error comes from the correlation with $L_{-}$at/L in Fig. 6 with real data. The results from the Monte Carlo simulations are interesting to understand the results but hamper the flow of the paper. I suggest to move this part to an Appendix.

Interactive comment on Atmos. Meas. Tech. Discuss., doi:10.5194/amt-2020-407, 2020. 